\title{
A successful rescue of juvenile fulminant myocarditis by extracorporeal membrane oxygenation combined with intra-aortic balloon pump: a case report
}

\author{
Feng Zhao", Guoning Shi", Zhenhua Wu, Zhigang Guo \\ Department of Cardiac Surgery, Tianjin Chest Hospital, Tianjin, China \\ \#These authors contributed equally to this work. \\ Correspondence to: Zhenhua Wu, MD; Zhigang Guo, MD. Department of Cardiac Surgery, Tianjin Chest Hospital, No. 261, Taierzhuangnan Road, \\ Jinnan District, Tianjin 300222, China. Email: wzh8306@aliyun.com; m15233622238@163.com.
}

\begin{abstract}
Myocarditis refers to myocardial inflammatory damage, and it can have various causes. Myocarditis can lead to impaired cardiac function, including decreased systolic and diastolic function and arrhythmia. When myocarditis occurs suddenly and rapidly progresses to severe heart failure, hypotension, cardiogenic shock, or severe arrhythmia, it can be diagnosed as fulminant myocarditis (FM). Our hospital recently admitted a case of juvenile FM, a 17-year-old male, who was admitted to the hospital for "intermittent chest tightness and asthma for 10 days". When the patient was hospitalized, he complained of chest tightness, palpitation, shortness of breath, fatigue, and dyspnea. Wet rales could be heard in both lungs on auscultation, as well as arrhythmia. Echocardiograms indicated an ejection fraction of $10 \%$. Laboratory tests showed liver and kidney dysfunction as well as abnormal coagulation function. After admission, he was treated with drugs including cardiotonic, diuretic, hormonal, antiviral drugs, and drugs affecting immune regulation. After tracheal intubation-assisted ventilation, the patient's symptoms did not significantly alleviate, and then veno-arterial extracorporeal membrane oxygenation (V-A ECMO) combined with an intra-aortic balloon pump (IABP) were used to assist in the treatment of cardiopulmonary failure. After the treatment, the patient's hemodynamics were stable, the respiratory and circulatory functions were restored, and the ECMO and IABP were successfully removed. The patient's review results were satisfactory, and he was discharged without delay. Although ECMO has no direct therapeutic effect on myocarditis, it can quickly stabilize hemodynamics, improve systemic tissue perfusion, reduce the use of high-dose vasoactive drugs and effectively assist patients during the acute phase of myocarditis. The authors believe that ECMO is the first choice for the treatment of FM in adolescents. Here we summarize our experience at our hospital with the treatment of FM using life support methods.
\end{abstract}

Keywords: Fulminant myocarditis (FM); extracorporeal membrane oxygenation (ECMO); intra-aortic balloon pump (IABP); case report

Submitted May 20, 2021. Accepted for publication Aug 16, 2021.

doi: $10.21037 /$ atm-21-3959

View this article at: https://dx.doi.org/10.21037/atm-21-3959

\section{Introduction}

Fulminant myocarditis (FM) is a systemic disease characterized by a severe myocardial limitation or diffuse inflammatory injury. Its clinical features are rapid onset, the rapid emergence of hemodynamic disorders that are difficult to correct, and severe heart failure, malignant arrhythmia, cardiogenic shock, and even multiple organ failure (1). FM frequently occurs in young and middle-aged people with acute onset and rapid progress, and the early mortality rate is as high as $50 \%$ (2). In 2017, Ammirati et al. further updated the FM diagnostic criteria: acute onset (less than 2 to 4 weeks since its initial symptom); cardiogenic shock 
or arrhythmia leads to hemodynamic instability or even sudden death; it needs hemodynamic support, cardiotonic and (or) mechanical circulation assisted device; histological examination regardless of the type of inflammatory infiltration is multifocal active myocarditis (3). Although FM is a dangerous condition, once the patient has passed the acute stage, the condition tends to be stable, and the prognosis is generally good (4). How to help FM patients survive the acute phase safely is the focus of treatment for such patients. FM has no specific drug treatment, and the conservative treatment effect of medical drugs is poor; hence, it is often necessary to insert mechanical auxiliary devices to support the treatment. A multi-center study showed that adopting a "comprehensive treatment plan based on life support" including mechanical life support, immunomodulatory therapy and antiviral therapy (especially neuraminidase inhibitors) can reduce in-hospital mortality rate of patients of FM to below 5\% (5). Recently, a juvenile FM patient with severe heart failure and liver and kidney insufficiency was treated with extracorporeal membrane oxygenation (ECMO) combined with an intraaortic balloon pump (IABP). Following this, the auxiliary machine was successfully removed from the patient, and the patient was discharged. There have been many successful cases of using ECMO combined with IABP to treat adult FM. The author's team is the first to use this method for the treatment of juvenile FM, which has good efficacy in therapy. This paper reports this case and summarizes some experiences at our hospital in treating FM with ECMO combined with IABP.

We present the following case in accordance with the CARE reporting checklist (available at https://dx.doi. org/10.21037/atm-21-3959).

\section{Case presentation}

The patient, male, aged 17 years, was admitted to the hospital mainly because of "intermittent chest tightness and asthma for 10 days". Before that, the patient was in good health. There were no obvious precursors during the 10 days before admission, such as chest tightness, dyspnea, fatigue, poor appetite, or cough. Symptoms appeared intermittently, aggravated gradually, and dyspnea occurred. He was admitted to our hospital for emergency treatment. The physical examination at admission showed: a body temperature of $36.3^{\circ} \mathrm{C}$, breathing 21 times/min, a heart rate of 101 times/min, and blood pressure of 103/65 mmHg. Blister sound and arrhythmia could be heard by auscultation in both lungs, and pathological murmur could be heard in each valve auscultation area. After admission, urgent laboratory tests were undertaken. Echocardiography showed that the ejection fraction was $10 \%$, that the left ventricle was enlarged, that a strong echo could be seen at the apex of the left ventricular wall, a thrombus was considered, and a small amount of pericardial effusion was observed. An electrocardiogram suggested sinus tachycardia. The following laboratory results were obtained: creatine kinase: 3,745.00 U/L, creatine kinase isoenzyme: $107.00 \mathrm{U} / \mathrm{L}$, hypersensitivity troponin T: $0.06 \mathrm{ng} / \mathrm{mL}$, B-type natriuretic peptide: $1,512.26 \mathrm{pg} / \mathrm{mL}$, alanine aminotransferase: 4,048.50 U/L, aspartate aminotransferase: 5,320.80 U/L, total bilirubin: $40.60 \mu \mathrm{mol} / \mathrm{L}$, creatinine: $112.00 \mu \mathrm{mol} / \mathrm{L}$, urea: $10.90 \mathrm{mmol} / \mathrm{L}$, uric acid: $804.00 \mu \mathrm{mol} / \mathrm{L}$, white blood cell count: $18.45 \times 10^{9} / \mathrm{L}$, neutrophil ratio: $82.5 \%$, lymphocyte ratio: $11.8 \%$, C-reactive protein: $13.10 \mathrm{mg} / \mathrm{L}$, platelet count: $121.00 \times 10^{9} / \mathrm{L}$, prothrombin time: $33.50 \mathrm{~s}$, international normalized ratio (INR) value: 3.12 , and fibrinogen quantity: $0.76 \mathrm{~g} / \mathrm{L}$. Diagnoses at admission were: FM, heart failure [New York Heart Association (NYHA) IV grade], arrhythmia, hepatic and renal insufficiency, and abnormal coagulation function. The treatment process was as follows:

(I) After being admitted to the hospital, the patient was treated with cardiotonic, diuretic, hormonal, antiviral, and other drugs and assisted ventilation without ventilator. The patient's symptoms were not significantly relieved, shortness of breath was aggravated, and his blood pressure continued to drop. Immediately after sedation and analgesia, endotracheal intubation and mechanical ventilation assisted breathing. An urgent examination of bedside ultrasound showed that the left ventricular ejection fraction (LVEF) was 10\%, the left heart was enlarged, and the left ventricular wall motion was widely decreased, and hence ECMO support treatment was performed. The mode was a femoral artery-femoral vein $(\mathrm{V}-\mathrm{A})$ bypass, and the flow rate was maintained at $3.0-3.5 \mathrm{~L} / \mathrm{min}$.

(II) After 6 hours of ECMO adjuvant therapy, bedside ultrasound was reexamined, which indicated that the left ventricle was still distended, and the systolic function had not markedly recovered. Therefore, IABP adjuvant therapy was performed using the right femoral artery to reduce the cardiac afterload and increase the myocardial oxygen supply. At the same time, the patient was given antibiotics to 
prevent infection, interferon to protect against viral infection, glucocorticoids and immunoglobulins to regulate immunity, and trimetazidine, creatine phosphate, and coenzyme Q to protect and sustain the myocardium.

(III) After 10 days of ECMO combined with IABP, the patient's hemodynamics were stable, the echocardiogram showed recovery of left ventricular systolic function, and theLVEF was reestablished to $30 \%$. At reexamination, the laboratory values were as follows: B-type natriuretic peptide: $870.58 \mathrm{pg} / \mathrm{mL}$, alanine aminotransferase: $144.40 \mathrm{U} / \mathrm{L}$, aspartate aminotransferase: $41.20 \mathrm{U} / \mathrm{L}$, total bilirubin: $36.80 \mu \mathrm{mol} / \mathrm{L}$, urea: $8.40 \mathrm{mmol} / \mathrm{L}$, and uric acid: $98.00 \mu \mathrm{mol} / \mathrm{L}$. Laboratory tests showed that heart, liver, and kidney function were markedly better than those at admission and indicated recovery. The ECMO was removed, and the IABP and other supportive treatments were continued. Eight days later, the patient's breathing and circulation were stable. After removing the IABP and continuing other symptomatic treatment for 1 week, the patient was discharged uneventfully.

All procedures performed in studies involving human participants were in accordance with the ethical standards of the institutional and/or national research committee(s) and with the Helsinki Declaration (as revised in 2013). Written informed consent was obtained from the patient for publication of this case report and accompanying images. A copy of the written consent is available for review by the editorial office of this journal.

\section{Discussion}

FM is the most serious type of myocarditis. Patients show rapid-onset, abnormal hemodynamics, and severe arrhythmia, and these may be accompanied by respiratory, liver, and kidney failure. The early mortality rate is extremely high. Causes include infection, autoimmune diseases, and toxin/drug toxicity. These infections are the most important cause, and viruses are the most common pathogens, including enteroviruses (especially coxsackie B virus), and adeno-, cytomegalo-, Epstein-Barr, and influenza viruses (6). The main mechanism of myocardial injury is that the virus directly attacks myocardial cells and replicates in cells, causing myocardial degeneration, necrosis, and dysfunction. At the same time, the body produces cellular and humoral immune responses to viruses, and a large number of cytokines and inflammatory mediators released by infiltrating inflammatory cells and tissue cells can cause damage to the myocardium and entire organs and tissues (7). Some studies have found that the LVEF of FM patients is worse than that of patients with other types of myocarditis at admission. However, the LVEF of FM patients improves more markedly during hospitalization, indicating that FM patients' heart function has good reversibility with the support of effective circulation (3). Heart injury is the main cause of death of patients, and pump dysfunction caused by heart injury is the decisive factor of the severity of patients' illness, and hence mechanical support for heart pump function and circulation is the decisive factor of patients' prognosis (1).

The concept of ECMO originated from cardiopulmonary bypass (CPB); ECMO is a simplified $\mathrm{CPB}$ device that can be used for long periods. In recent years, ECMO technology has been widely used with good results in the emergency adjuvant treatment of acute circulatory and/or respiratory failure of various causes. When ECMO occurs, blood is drawn from the vein, oxygenated through a membrane lung, and carbon dioxide is discharged simultaneously. After the gas exchange, the blood is pumped back to a human vein $(\mathrm{V}-\mathrm{V}$ mode) or artery ( $\mathrm{V}$-A mode). Although ECMO has no direct therapeutic effect on myocarditis, it can quickly stabilize hemodynamics, correct the deterioration of the circulatory system, improve the perfusion of wholebody tissues, reduce the use of large doses of vasoactive drugs, avoid the occurrence of multiple organ dysfunction syndromes (MODS), and buy time for the recovery of the heart. It is the first choice for the treatment of FM. The case reported here concerns a minor patient and where the diagnosis of FM is clear. Since the patient is a minor, the significance and value of treatment are significant. After admission, ECMO combined with IABP support treatment has successfully helped the patient survive the FM acute phase.

The medical treatments of FM include antiviral agents, glucocorticoids combined with gamma globulin, vasoactive drugs, etc. However, due to the rapid onset and dangerous progression of FM, it is often accompanied by severe arrhythmia and refractory heart failure, such that the response to medical treatment is often poor. Therefore, life support therapy is the preferred treatment approach for FM. During FM, the myocardium, including the pump function, is extensively and severely damaged. In addition to the damage due to pulmonary inflammation, systemic respiratory and circulatory failure occurs. During life 
support, the heart can rest, and subsequently, heart function can be gradually restored, and systemic conditions can be improved using systemic treatment. Here is a summary of the experiences made at our hospital using life support methods to treat FM:

(I) Grasping the opportunity and starting ECMO treatment in time is the key to improve the success rate of FM treatment. ECMO support is suitable for advanced cases that are often complicated with multiple organ failure, where the response to treatment effect is not good. FM patients can rapidly develop pump failure, which leads to cardiogenic shock, malignant arrhythmia, and even sudden death, and at the same time, they are prone to multiple organ failure, which increases the risk of death. Therefore, the authors also believe that ECMO should be started sooner rather than later when workforce, material resources, and financial resources permit, especially when combined with multiple organ dysfunction, to prevent MODS. Patients in the following situations should start ECMO as soon as possible: patients with severe arrhythmia such as ventricular fibrillation, cardiac arrest, short-term ventricular tachycardia, or third-degree atrioventricular block, who cannot maintain effective circulation even after having been treated with antiarrhythmic drugs; patients who are given two or more positive inotropic drugs/vasoactive drugs and still have hypotension even after large doses have been administered; echocardiography indicates that the patient has diffusely decreased wall motion, an LVEF $<40 \%$ and a heart index $<2 \mathrm{~L} /\left(\mathrm{m}^{2} \cdot \mathrm{min}\right)$; arterial blood gas analysis shows that the patient has severe metabolic acidosis and lactic acid $>2.0 \mathrm{mmol} / \mathrm{L}$ and shows progressive aggravation; and finally patients with multiple manifestations of other tissues and organs, including abnormal liver function, renal function damage, abnormal coagulation function, and severe lung damage, etc.

(II) Standardized management of ECMO during treatment is an important factor affecting the therapeutic effect. Ensure adequate flow at an early stage, stabilize vital signs, reduce heart load, repay oxygen debt, correct internal environment imbalance, and gradually reduce or stop vasoactive drugs. Ensure a stable internal environment in the medium term, wait for the recovery of cardiac function and prevent complications. At more advanced stages, gradually reduce the flow rate, increase the work done by the autologous heart, appropriately adjust the ventilator parameters and positive inotropic drugs, and evaluate the patient's vital signs, hemodynamic conditions, and cardiac function indicators to prepare for the removal of the auxiliary machines.

(III) ECMO combined with non-drug therapy can effectively reduce the incidence of MODS and improve the prognosis of patients. (i) Ventilatorassisted ventilation can effectively improve lung function and reduce the work done by the patient's heart. It is an important treatment for FM complicated with left heart failure, and it is recommended to use it as soon as possible. Ventilator-assisted ventilation without a planer is only suitable for conscious patients who cooperate with ventilator ventilation. If the patient has shortness of breath or difficulty breathing, respiratory failure, and in particular obvious respiratory and metabolic acidosis that affects consciousness, it should be changed to endotracheal intubation as soon as possible. (ii) If the patient still has clinical manifestations such as oliguria, anuria, volume overload, and hyperkalemia while receiving effective ECMO flow perfusion, continuous renal replacement therapies (CRRT) should be used as early as possible. Its main functions are: clearing away various small molecular toxins and inflammatory mediators, downregulating inflammatory reactions and reducing organ damage; correcting the disturbance of water, electrolyte, and acid-base balance and maintaining the stability of the internal environment; effectively reducing tissue edema, as well as improving tissue oxygen supply and organ function. In addition, to effectively remove toxic substances, CRRT needs to be continuously carried out every day for at least 8-12 hours or longer, and the process of blood drawing and returning must be slow to avoid inducing circulatory failure. (iii) When the patient suffers from a severe decrease of myocardial contractility or myocardial stunning, and ultrasound indicates that the left ventricular wall motion is widely reduced. The aortic valve is incomplete, IABP is used in combination to reduce cardiac afterload and cardiac work, increase stroke 
volume, increase circulatory perfusion of important organs such as the heart and brain, and reduce the use of vasoactive drugs, to help patients successfully pass through the acute phase of FM.

(IV) Actively adopting various treatment strategies to prevent ECMO-related complications can effectively improve the outcome of patients. Bleeding, ischemia and necrosis of lower limbs, and infection are common complications of ECMO treatment. (i) Because heparinized anticoagulation is needed during ECMO treatment, and very ill patients often have an abnormal liver function, patients may bleed at the catheter. Our approach is to inject platelets, plasma, human fibrinogen, blood coagulation factor, etc., to correct the coagulation disorder while maintaining a slightly lower activated clotting time (ACT) level (<180 s) and to monitor the coagulation function and platelet status of patients every day. (ii) Given the possible ischemic necrosis of the lower limbs following femoral artery intubation, vascular ultrasound should be used to evaluate the thickness of the femoral artery before intubation, and an appropriate type of arterial intubation should be selected. The subclavian artery can be selected for catheterization for patients with a narrow femoral artery or calcified lesions. Secondly, following conventional femoral artery intubation, selecting an appropriate side branch circulation line for distal perfusion intubation can effectively prevent lower limb ischemia. (iii) To prevent the occurrence of infection, ECMO should be established under strictly aseptic conditions, good hand hygiene should be practiced, surfaces should be disinfected daily to avoid cross-infection in the hospital, and various infection indicators should be closely monitored. Once the infection has occurred, antibiotics should be used as soon as possible for symptomatic treatment. During ECMO establishment, if the incision exposure time is long, wound infection and delayed healing can easily occur following the stopping of the ECMO. Our experience is that after stopping the ECMO, if local debridement of the wound is routinely performed, drainage tubes are placed if necessary, adipose tissue is carefully ligated to prevent lymphatic fistulas, and wound closure is achieved layer by layer; this can effectively prevent wound infection and delayed healing.
(V) The development of ECMO technology is complicated and risky and often requires many medical resources. To make ECMO treatment safe, economical, and effective, it is absolutely necessary to establish a professional, cooperative, efficient, and responsible ECMO team.

In summary, ECMO can quickly improve the hemodynamic stability of FM patients, effectively help patients survive the acute phase and improve the prognosis. Clinicians should predict the trend of FM patients' illness in the context of the trajectory of the condition and use ECMO and other non-drug treatment methods for life support treatment as soon as possible before the illness worsens. Patients should be closely monitored to prevent complications, improve treatment success, and save patients' lives.

\section{Limitation}

There are no similar cases that can be used for controlled studies. We will continue to collect data on similar cases.

\section{Acknowledgments}

Funding: This work was supported by Science and Technology Bureau of Jinnan District, Tianjin (No. 20200115).

\section{Footnote}

Reporting Checklist: The authors have completed the CARE reporting checklist. Available at https://dx.doi. org/10.21037/atm-21-3959

Conflicts of Interest: All authors have completed the ICMJE uniform disclosure form (available at https://dx.doi. org/10.21037/atm-21-3959). Dr. FZ reports that this work was supported by Science and Technology Bureau of Jinnan District, Tianjin [No. 20200115: Transformation and Application of ECMO "Tianjin Chest Hospital Model" in the "New Coronavirus Pneumonia" Epidemic and Research and Development of Key Technologies]. The other authors have no conflicts of interest to declare.

Ethical Statement: The authors are accountable for all aspects of the work in ensuring that questions related to the accuracy or integrity of any part of the work are appropriately investigated and resolved. All procedures 
performed in studies involving human participants were in accordance with the ethical standards of the institutional and/or national research committee(s) and with the Helsinki Declaration (as revised in 2013). Written informed consent was obtained from the patient for publication of this case report and accompanying images. A copy of the written consent is available for review by the editorial office of this journal.

Open Access Statement: This is an Open Access article distributed in accordance with the Creative Commons Attribution-NonCommercial-NoDerivs 4.0 International License (CC BY-NC-ND 4.0), which permits the noncommercial replication and distribution of the article with the strict proviso that no changes or edits are made and the original work is properly cited (including links to both the formal publication through the relevant DOI and the license). See: https://creativecommons.org/licenses/by-nc-nd/4.0/.

\section{References}

1. Kociol RD, Cooper LT, Fang JC, et al. Recognition and initial management of fulminant myocarditis: a scientific statement from the American Heart Association. Circulation 2020;141:e69-92.

2. Edin ML, Zeldin DC. An improved protocol for the

Cite this article as: Zhao F, Shi G, Wu Z, Guo Z. A successful rescue of juvenile fulminant myocarditis by extracorporeal membrane oxygenation combined with intra-aortic balloon pump: a case report. Ann Transl Med 2021;9(16):1355. doi: 10.21037/atm-21-3959 treatment of fulminant myocarditis. Sci China Life Sci 2019;62:433-4.

3. Ammirati E, Cipriani M, Lilliu M, et al. Survival and left ventricular function changes in fulminant versus nonfulminant acute myocarditis. Circulation 2017;136:529-45.

4. McCarthy RE 3rd, Boehmer JP, Hruban RH, et al. Longterm outcome of fulminant myocarditis as compared with acute (nonfulminant) myocarditis. $\mathrm{N}$ Engl J Med 2000;342:690-5.

5. Li S, Xu S, Li C, et al. A life support-based comprehensive treatment regimen dramatically lowers the in-hospital mortality of patients with fulminant myocarditis: a multiple center study. Sci China Life Sci 2019;62:369-80.

6. Veronese G, Ammirati E, Brambatti M, et al. Viral genome search in myocardium of patients with fulminant myocarditis. Eur J Heart Fail 2020;22:1277-80.

7. Caforio AL, Pankuweit S, Arbustini E, et al. Current state of knowledge on aetiology, diagnosis, management, and therapy of myocarditis: a position statement of the European Society of Cardiology Working Group on Myocardial and Pericardial Diseases. Eur Heart J 2013;34:2636-48, 2648a-2648d.

(English Language Editors: B. Meiser and J. Chapnick) 